\title{
Nuclear theory nudged
}

For 40 years, physicists at the Oak Ridge Electron Linear Accelerator (ORELA) at Oak Ridge National Laboratory in Tennessee fired bursts of neutrons at various targets to probe the structure of atomic nuclei. Now, with the facility effectively mothballed by a shortage of funds, a newly published result based on data gathered at ORELA has challenged a well-established theory of the nucleus. Independent experts say that further measurements should be made to follow up the tantalizing claim, which would involve putting the facility back on line.

In 2002, Oak Ridge physicist Paul Koehler and his colleagues used the neutron beam to measure 'neutron resonances' in each of four different isotopes of platinum. The resonances are particular energies at which the neutrons are especially likely to be absorbed by the platinum nuclei. The motion of protons and neutrons inside the platinum nuclei affects the pattern of resonances. And according to random matrix theory, a mathematical theory that for decades has been crucial for calculating the behaviour of large nuclei, those motions should be chaotic.

Yet, as Koehler and his colleagues report this month in Physical Review Letters (P. E. Koehler et al. Phys. Rev. Lett. 105, 072502; 2010), their analysis of the ORELA data found no sign that the nucleons in platinum were moving chaotically. By looking at the strength of the resonances, rather than just their spac-

“There's a lot of good physics we could do if we could run." ing, the group rejects the applicability of random matrix theory with a $99.997 \%$ probability. Instead, the nucleons seem to move in a coordinated fashion. "There's no viable model of nuclear structure that could explain this," says Koehler.

The resolution of the puzzle could have practical implications, as random matrix theory is currently used to estimate the probability that escaping neutrons will collide with nuclei, and from this to calculate the amount of shielding needed in nuclear reactors and stockpiles. "Engineers build in some extra shielding to cover the uncertainty, but if you were building 100 nuclear reactors you'd want the precision," says Gary Mitchell of North Carolina State University in Raleigh, co-author of a recent review article on random matrix theory (H. A. Weidenmüller and G. E. Mitchell Rev. Mod. Phys. 81, 539; 2009).

Mitchell adds that further experiments on nuclei other than platinum are urgently needed to determine whether the theory has truly

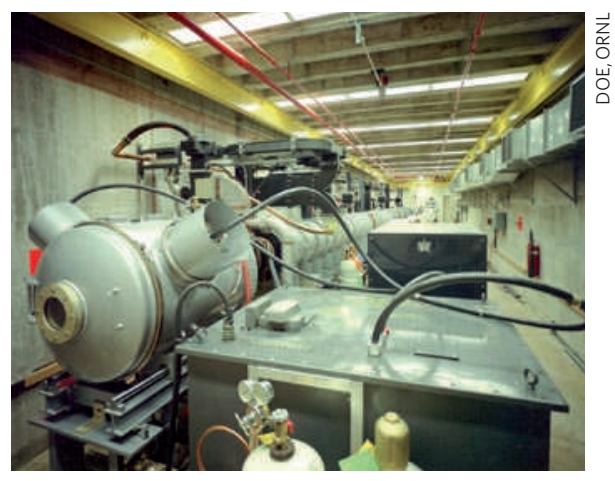

Fund-starved ORELA could still spring a surprise.

broken down in this experiment, as Koehler and colleagues suggest, or whether - as Mitchell suspects - the shape of the platinum nucleus is unusual in some way that would account for the result.

Oriol Bohigas of the University of ParisSouth, a leader in the field of random matrix theory, also recommends additional measurements. He says Koehler and his colleagues need to repeat measurements made at Columbia University in New York in the 1970s, which first contributed to the theory's acceptance, in order to see whether modern instruments and data-analysis methods give the same results.

But additional measurements at ORELA seem unlikely. "It hasn't been adequately funded and it's on standby," says Jim

Beene, director of the Physics Division at Oak Ridge National Laboratory. According to the US Department of Energy, Koehler and Beene received US $\$ 105,998$ from the department's Office of Science for ORELA operations in fiscal year 2009, only about a tenth of what the researchers have said would be needed to run it. Beene explains that with the demise of the US fast-reactor programme in the 1980s, direct funding for ORELA dried up; the energy department says that other research efforts are a higher priority for the nuclear-science community.

According to Koehler, the only other place in the world where similar measurements could be made is at the Geel Electron Linear Accelerator (GELINA) at Geel in Belgium, which has a flux, energy resolution and source brightness to rival ORELA. But, Koehler says, he'd love to take additional measurements himself. "There's a lot of good physics we could do if we could run," he says.

Eugenie Samuel Reich 